\title{
Hypersensitivity to amphetamine's psychomotor and reinforcing effects in serotonin transporter knockout rats: glutamate in the nucleus accumbens
}

\author{
Lucia Caffino $^{1}$, Michel Verheij ${ }^{2}$, Karine Roversi ${ }^{2}$, Giorgia Targa ${ }^{1}$, Francesca Mottarlini ${ }^{1}$, \\ Piotr Popik ${ }^{3}$, Agnieszka Nikiforuk ${ }^{3}$, Joanna Golebiowska ${ }^{3}$, Fabio Fumagalli ${ }^{1}$, and Judith \\ Homberg $^{2}$ \\ ${ }^{1}$ University of Milan \\ ${ }^{2}$ Radboud Universiteit Nijmegen Donders Institute for Brain Cognition and Behaviour \\ ${ }^{3}$ Institute of Pharmacology of the Polish Academy of Sciences
}

April 28, 2020

\begin{abstract}
Background and purpose: Amphetamine use disorder is a serious health concern, but surprisingly little is known about the vulnerability to the moderate and compulsive use of this psychostimulant and its underlying mechanisms. Previous research showed that inherited serotonin transporter (SERT) down-regulation increases the motor response to cocaine, as well as moderate and compulsive intake of this psychostimulant. Here we sought to investigate whether these findings generalize to amphetamine and the underlying mechanisms in the nucleus accumbens. Experimental Approach: In serotonin transporter knockout (SERT/-) and wild-type control (SERT+/+) rats we assessed the locomotor response to acute amphetamine (AMPH) and intravenous AMPH self-administration under short access (ShA: 1 hr daily sessions) and long access (LgA: 6 hr daily sessions) conditions. $24 \mathrm{hrs}$ after AMPH self-administration we analysed the expression of glutamate system components in the nucleus accumbens shell and core. Key results: We found that SERT-/- animals displayed an increased AMPH-induced locomotor response and increased AMPH self-administration under LgA, but not ShA conditions. Further, we observed changes in the vesicular and glial glutamate transporters, NMDA and AMPA receptor subunits and their respective postsynaptic scaffolding proteins as function of serotonin transporter genotype, AMPH exposure (baseline, ShA and LgA) and nucleus accumbens sub region. Conclusion and implications: We demonstrate that SERT gene deletion increases the psychomotor and reinforcing effects of AMPH, and that the latter is potentially mediated, at least in part, by homeostatic changes in the glutamatergic synapse of the nucleus accumbens shell and/or core.
\end{abstract}

\section{Bullet point summary}

What is already known:

Rats lacking the serotonin transporter show increased moderate and compulsive cocaine self-administration.

What this study adds:

- Serotonin transporter knockout rats show also increased compulsive, but not moderate, amphetamine self-administration.

- This is associated with glutamatergic synaptic changes in the nucleus accumbens shell and core.

\section{Clinical significance:}


We provide a mechanism for amphetamine addiction proneness in individuals with inherited serotonin transporter down-regulation.

\section{Introduction}

Amphetamine (AMPH) is a psychostimulant substance widely used and with growing expansion in the entire world because of its euphoric effects (World Drug Report, 2018). One in six psychostimulant users develops dependency on the drug (Wagner \& Anthony, 2002). However, up to date, it is not clear which factors shape vulnerability to AMPH dependency and the underlying mechanisms are still elusive. This would be critical as such insight ultimately would help to inform early interventions or treatments.

In humans, the short (s) low expression allelic variant of the serotonin transporter-linked polymorphic region (5-HTTLPR) has been considered as risk allele for dependency on MDMA (ecstasy) and cocaine (Enoch et al., 2011; Gerra et al., 2007; Mannelli et al., 2005; Martin-Santos et al., 2010). Accordingly, we and others previously observed that serotonin transporter knockout (SERT ${ }^{-/}$) rats, which model the 5-HTTLPR s-allele (Holmes et al., 2010; Schipper et al., 2019) in humans, are more sensitive to the to the psychomotor and reinforcing effects of these psychostimulants (Homberg et al., 2008; Oakly et al., 2014; Pettie et al., 2019; Verheij et al., 2018). However, as to whether these observations generalize to amphetamine is currently not known.

Mechanistically, psychostimulants increase extracellular levels of dopamine, serotonin and noradrenaline via an action on their respective transporter, although with varying sensitivity for the different psychostimulants. In particular, cocaine and AMPH are thought to mediate their reward-related effects by increasing dopamine levels (Koob, 1992; Natarajan \& Yamamoto, 2011; Rocha et al., 1998; Rothman \& Glowa, 1995; Woolverton \& Johnson, 1992; Lile \& Nader, 2003). Yet, we observed that cocaine, more strongly increased locomotor activity in $\mathrm{SERT}^{-/-}$versus their wild-type $\mathrm{SERT}^{+/+}$counterparts (Homberg et al., 2008). In addition, the cocaine-induced increase of extracellular serotonin, but not of extracellular dopamine and noradrenaline, was reduced in the nucleus accumbens (NAc) of $\mathrm{SERT}^{-/}$-rats (Verheij et al., 2014). This suggests that SERT and accumbal serotonin may play a critical role in the psychomotor and reinforcing effects of psychostimulants. Given the previously observed SERT-dependent changes in the psychomotor and reinforcing effects of cocaine, we have now analyzed the SERT dependent psychomotor and reinforcing effects of AMPH. To this end, the acute locomotor response to various doses of AMPH, as well as AMPH self-administration under short access (ShA, 1 h/day) conditions (modeling moderate drug use), and long access (LgA, 6 h/day) conditions (modeling uncontrolled compulsive drug use) (Ahmed \& Koob, 1998) has been obtained in SERT ${ }^{-/-}$and their wild-type control rats.

From a neurobiological point of view, the transition from goal-directed drug intake toward addictive states has been proposed to result from a shift of control of motivated behavior from the prefrontal cortex (PFC) to the striatum via glutamatergic projections (Everitt \& Robbins, 2016; Kalivas \& Volkow, 2005). We have recently shown that the interaction between serotonin and cocaine intake, as well as the transition from hedonic to compulsive use of the psychostimulant, dysregulates glutamatergic synapses in the habenula and PFC (Caffino et al., 2020; Caffino et al., 2019), pointing to serotonin-glutamate interactions as a neurobiological substrate of heightened vulnerability to drug dependence. Yet, surprisingly, little is known about the AMPH-induced neuroadaptations on glutamate signalling and the serotoninergic control on glutamatergic homeostasis in the transition from hedonic to compulsive AMPH use (Faraone, 2018). Interestingly, it has been demonstrated that AMPH reduced striatal protein expression of metabotropic mGluR5 receptor in rats (Shaffer et al., 2010), suggesting that altered glutamate signalling can contribute to altered sensitivity to the psychomotor and reinforcing effects of amphetamine.

The NAc is a brain region critical for the neuroadaptive changes sub-serving drug intake, withdrawal and motivation to search for drugs. This brain region is characterized by the above-mentioned SERT-dependent changes in the serotonin response to psychostimulants and a strong glutamatergic signalling. On this basis, we sought to determine the SERT-dependent effects of AMPH self-administration on the accumbal glutamatergic synapse. To this end, we deeply analyzed the glutamate synapse in the NAc. Specifically, because the NAc 
core subregion (cNAc) is selectively involved in incubation of drug seeking, whereas the NAc shell (sNAc) is involved in escalation of drug taking (Guillem et al., 2014), we investigated glutamate homeostasis in both subregions and focused on: 1) the mechanisms regulating the storage of glutamate in presynaptic vesicles, which is mediated by the vesicular glutamate transporter (vGlut1) (El Mestikawy et al., 2011), 2) the clearance of the neurotransmitter from the synaptic cleft by the glutamate transporter (GLT-1) (RobertsWolfe \& Kalivas, 2015), as well as 3) the main glutamate post-synaptic receptors (NMDA and AMPA) (Traynelis et al., 2010) and 4) the main scaffolding proteins of NMDA (SAP102) and AMPA (SAP97, GRIP) glutamate receptors, whose job is to firmly anchor these receptors to the membrane (Oliva et al., 2012).

\section{Materials and Methods}

Our studies have been executed according to ARRIVE guidelines (Kilkenny et al., 2010).

\section{Animals}

We employed rats in this study because the rat is the preferred species for preclinical addiction research (Homberg et al., 2017). We used SERT ${ }^{-/-}$rats as model for the human SERT gene polymorphism as has been established previously (Caspi et al., 2010). SERT ${ }^{-/-}$rats (SLC6A4 $4^{1 \mathrm{Hubr}}$ ) were generated by N-ethylN-nitrosurea (ENU) induced mutagenesis (Homberg et al., 2007; Smits et al., 2006) and outcrossed with commercially available Wistar rats (Harlan, Ter Horst, the Netherlands) for at least 15 generations. Male $\mathrm{SERT}^{-/}$and SERT ${ }^{+/+}$offspring were used for experiments as in human psychostimulant addiction has a clear male preponderance (Degenhardt et al., 2014). The rats were bred in Nijmegen and either tested in Nijmegen or transported to Poland. In Nijmegen, rats were housed in groups of 2 rats in enriched Macrolon type III cages ( $42 \times 26 \times 15 \mathrm{~cm}$; Techniplast 1291H, Tecnilab-BMI) with corncobs bedding (irradiated, SPPS COB12, Bio Services) under conventional conditions (no filtertops). The animals had access to food (dried pellets of standard chow food (Ssniff RM V1534-703 diet supplied by BioServices) and waterad libitum, except during test phases. The rats were housed under a reversed day and night cycle (lights off at 08:00 $\mathrm{AM})$ in temperature $\left(21 \pm 1^{\circ} \mathrm{C}\right)$ and humidity $(55 \pm 5 \%)$ controlled rooms. Testing (AMPH self-administration) took place in the dark phase of the light/dark cycle. In Poland, rats were group-housed 4 rats per cage (380 $\times 200 \times 590 \mathrm{~mm}$, Ehret Labor- und Pharmatechnik GMBH \& Co.KG, Germany) with aspen litter (MIDI LTE E-002 Abedd, AnimaLab, Poland) and environmental enrichment. The rats had free access to food (VRF1 (P) Special Diets Services, England) and water. Animals were housed in a temperature-controlled $(21+-1 \operatorname{deg} \mathrm{C})$ and humidity-controlled $(40-50 \%)$ colony room under a 12/12 h light/dark cycle (lights on at 06:00 AM). Behavioural testing (locomotor activity test) was performed during the light phase of the light/dark cycle. Testing was always done blindly by an experimenter who was unaware of the genotype of the animals. All procedures were carried out in agreement with the current Directive 2010/63/EU and Dutch and Polish Research Council Guide for the Care and Use of Laboratory Animals and were approved by local and national Institutional Animal Care and Use Committes. The experimental procedures at Radboudumc (Nijmegen, The Netherlands) were performed under a project license from the Central Committee on Animal Experiments (Centrale Commissie Dierproeven, CCD, The Hague, The Netherlands), in full compliance with the legal requirements of Dutch legislation on the use and protection of laboratory animals (Animal Testing Act, WOD). The experimental procedures in Poland were performed at the Maj Institute of Pharmacology, Polish Academy of Science, Krakow, Poland. All efforts were made to reduce the number of animals used and their suffering both in the Netherlands and in Poland.

\section{Drugs}

Dex(d)-Amphetamine was provided by Spruyt Hillen (IJsselstein, the Netherlands) and was dissolved in saline $0,9 \%$.

\section{Behaviour}

\section{AMPHETAMINE-induced locomotor activity}

The psychomotor effects of AMPH were tested in a grey plywood open field of $50 \times 50 \times 50 \mathrm{~cm}$, which was 
illuminated by 20 Lux. After 30 mins of habituation, rats were treated with $0.5 \mathrm{mg} / \mathrm{kg}$ amphetamine $\left(\mathrm{SERT}^{+/+}: \mathrm{n}=12 ; \mathrm{SERT}^{-/-}: \mathrm{n}=12\right), 1 \mathrm{mg} / \mathrm{kg}$ amphetamine $\left(\mathrm{SERT}^{+/+}: \mathrm{n}=13\right.$; $\mathrm{SERT}^{-/-}: \mathrm{n}=13$ ) or vehicle $\left(\mathrm{SERT}^{+/+}: \mathrm{n}=12\right.$; vehicle SERT ${ }^{-/-}: \mathrm{n}=12$ ) according to a standard Latin square design using the same animals with a break of 4 weeks between the various treatments. Distance moved was recorder for 120 mins (Fig 1a), and analyzed afterwards using the ANY-maze video tracking system (version 4.82) from Stoelting.

\section{Amphetamine self-administration}

\section{Intravenous catheterization}

Under isoflurane aesthesia, SERT ${ }^{+/+}$and $\mathrm{SERT}^{-/-}$rats, weighing between 300 and $450 \mathrm{~g}$, received a micro Renathane catheter into the right external jugular vein (for details, see: (Wee et al., 2007). The tubing (0.037" o.d. x 0.023" i.d., Braintree Scientific Inc., USA) was guided subcutaneously to a stainless-steel cannula (22G, Plastics One Inc., USA) at the back of the rat. After surgery, rats were given Flunixin (analgesic drug: $2.5 \mathrm{mg} / \mathrm{ml} / \mathrm{kg}$, s.c.) and Cefazolin (antibiotic drug: $15 \mathrm{mg} / \mathrm{ml} / \mathrm{kg}$ ) daily for respectively 3 and 7 days. After surgery, rats were singly housed to prevent damage to the cannula due to cage mates. These rats received a wooden block and nest material as cage enrichment, but not a shelter to prevent collisions with the cannula. The body weight of the animals was monitored daily and full recovery of bodyweight happened within 3 to 5 days. During recovery, no unexpected changes in animal behavior (e.g. no self-grooming, no water intake) were observed. Catheter patency was maintained by daily flushing with $0.2 \mathrm{ml}$ heparinized saline. In case of obstruction of the catheter, the animal was removed from the study.

\section{Self-administration chambers}

After, at least, one-week of recovery, AMPH self-administration was performed in standard operant boxes ( $28 \times 26 \times 20 \mathrm{~cm}$, Med Associates Inc., USA). The cannula was connected to a swivel system that allowed the animal to move freely. Once the animals were connected to the system, 2 retractable levers were presented. Pressing the active (correct) lever resulted in i.v. infusion of AMPH (volume: $0.1 \mathrm{ml}$ in $3 \mathrm{~s}$ ). Furthermore, a light cue above the active lever indicated a timeout period of $20 \mathrm{~s}$ during which no AMPH infusion was available. Pressing the inactive (incorrect) lever had no programmed consequences.

\section{Intravenous amphetamine self-administration training}

Rats were exposed to 1-hour daily training sessions $(0.03 \mathrm{mg} / \mathrm{kg} /$ infusion$)$ under a fixed ratio 1 (FR1) schedule over the course of 10 days. During training, the maximum number of infusions was set at 20 to prevent an early overdose.

\section{Short-access and long-access amphetamine self-administration}

After training, rats were divided into 2 groups with an equal number of AMPH injections during the last 3 training session. One group continued to self-administer AMPH $(0.03 \mathrm{mg} / \mathrm{kg} /$ infusion) for 1 hour/day (the short (ShA) access group: SERT ${ }^{+/+} \mathrm{n}=14, \mathrm{SERT}^{-/-} \mathrm{n}=13$ ), and the other group self-administered the same dose for 6 hours/day (the long (LgA) access group: $\mathrm{SERT}^{+/+} \mathrm{n}=14, \mathrm{SERT}^{-/} \mathrm{n}=13$ ) [see also: (Gipson \& Bardo, 2009)]. Protein expression data of these groups were compared to a new group of animals that was subjected to surgery, but were not exposed to AMPH (the AMPH-naive group: SERT ${ }^{+/+} \mathrm{n}=6$, $\mathrm{SERT}^{-/}$ $\mathrm{n}=6$; Fig 1b). The animals were tested daily between 8 am and $6 \mathrm{pm}$, for 18 consecutive days [see also: (Verheij et al., 2018)] and the order of testing ShA and LgA groups was changed every day. Due to a limited number of self-administration chambers, self-administration was performed in 2 cohorts of $\mathrm{SERT}^{+/+}$and SERT $^{-/-}$animals, of which the intake did not differ.

\section{Collection of nucleus accumbens tissue}

Twenty-four hours after the last ShA or LgA session, rats were decapitated without anesthesia, brains were collected, freshly frozen and stored at -80degC. Nucleus accumbens core (cNAc) and shell (sNAc) punches were collected from $220 \mathrm{um}$ brain slices, as previously described (Giannotti et al., 2016). The punches were collected according to coordinates as described by Paxinos and Watson (from Bregma +2.76 to Bregma 
$+0.84 \mathrm{~mm}$ ) (Paxinos and Watson, 2007), using a punching needle with a diameter of $1 \mathrm{~mm}$ (Fig 1c). For both regions, the two hemispheres were pooled. Punches were stored at -80degC.

\section{Protein extraction and Western blot analyses}

Bilateral punches of cNAc and sNAc of 63 animals were sonicated using a cold buffer containing $0.32 \mathrm{M}$ sucrose, $1 \mathrm{mM}$ Hepes solution, $0.1 \mathrm{mM}$ EGTA, $0.1 \mathrm{mM}$ PMSF, $\mathrm{pH}=7.4$, in presence of a complete set of protease inhibitors and a phosphatase inhibitor cocktail. Total proteins have been measured in the whole homogenate by the Bio-Rad Protein Assay (Bio-Rad Laboratories). Western blots were run as previously described (Caffino et al. 2016). Briefly, ten micro-grams of proteins for each sample were run on a sodium dodecyl sulfate- $8 \%$ polyacrylamide gel under reducing conditions and then electrophoretically transferred onto nitrocellulose membranes (GE Healthcare, Milan, Italy). Blots were blocked $1 \mathrm{~h}$ at room temperature with I-Block solution (Life Technologies Italia, Italy) in TBS $+0.1 \%$ Tween-20 buffer and then incubated with antibodies against the total proteins of interest.

The conditions of the primary antibodies were the following:

Anti vGlut1 (1:1000, Cell Signaling Technology Inc., RRID: AB_2797887), anti GLT1 (1:5000, AbCam, RRID: AB_1566262), anti GluN1 (1:1000, Invitrogen, RRID: AB_2533060), anti GluN2B (1:1000, Santa Cruz Biotechonology, RRID: AB_670229), anti GluN2A (1:1000, Invitrogen, RRID: AB_2536209), anti SAP102 (1:1000, Cell Signaling Technology Inc.), anti GluA1 (1:2000, Cell Signaling Technology Inc, RRID: AB_641040), anti GluA2 (1:2000, Cell Signaling Technology Inc., RRID: AB_10622024), anti SAP97 (1:1000, AbCam, RRID: AB_2091910), anti GRIP (1:2000, Synaptic system, RRID: AB_887728) and anti $\beta$-Actin (1:10000, Sigma-Aldrich, RRID: AB_476697).

Results were standardized using $\beta$-actin as the control protein, which was detected by evaluating the band density at $43 \mathrm{kDa}$. Immunocomplexes were visualized by chemiluminescence using the Chemidoc MP Imaging System (Bio-Rad Laboratories). Gels were run 2 times each and the results represent the average from 2 different runs.

\section{Data and Statistical Analysis}

The data and statistical analysis comply with the recommendations on experimental design and analysis in pharmacology (Curtis et al., 2018). Previous self-administration studies in cocaine-treated SERT S $^{-/}$and $\mathrm{SERT}^{+/+}$animals have revealed a significant genotype $\mathrm{x}$ self-administration session effect with an effect-size of about 40\% (see a.o. (Verheij et al., 2018)). Entering this effect size (based on the previously obtained standard deviation), together with an alpha-value of 0.05 and a power of 0.80 , into $\mathrm{G}^{*}$ power (version 3.1), revealed a minimum number of 13 to 14 rats per genotype. Because of the substantially smaller standard deviation typically observed in our molecular studies (e.g. Caffino et al., 2020), the number of animals in the AMPH-naive groups that were not exposed to self-administration were reduced to 6 .

\section{Behavioural data}

To analyze the AMPH -induced psychomotor responses, a three-way ANOVA with the factors genotype, AMPH dose and time (for repeated measures) was used. To analyze the AMPH self-administration data, number of rewards were analyzed using a three-way ANOVA with the factor genotype, access type and selfadministration session (for repeated measures). No outlier test was performed. Statistics were performed in IBM SPSS statistics version 23. Student's t-test was conducted as post-hoc test when allowed.

\section{Molecular data}

Molecular changes produced by genotype and AMPH exposure alone as well as by their combination were analyzed using a two-way ANOVA, with the factors genotype and type of AMPH access as independent variables. When dictated by relevant interaction terms, Tukey's multiple comparisons test was used to characterize differences among individual groups of rats. To enable visual comparisons across genotypes with different degrees of expression of glutamatergic molecular determinants, values are presented as percentage of the AMPH -naïve SERT ${ }^{+/+}$control rats that were not exposed to either AMPH ShA or LgA. Subjects 
were eliminated from the final dataset if their data deviated from the mean by 2 SDs. Prism 6.0 (GraphPad) was used to analyze all the data. Data are shown as mean \pm S.E.M and as $\%$ of baseline to control for unwanted sources of variation. Significance for all tests was assumed at $p<0.05$.

\section{Results}

\section{The psychomotor effects of amphetamine in $\mathrm{SERT}^{+/+}$and $\mathrm{SERT}^{-/-}$rats.}

AMPH resulted in a dose-dependent increase in locomotor activity over time (Fig 2: amphetamine dose $\mathrm{x}$ time effect: $\left.\mathrm{F}_{(8,372)}=36.688, \mathrm{p}<0.05\right)$. This increase in locomotor activity was stronger in $\mathrm{SERT}^{-/-}$compared to $\mathrm{SERT}^{+/+}$rats (Fig 2: genotype $\mathrm{x}$ amphetamine dose $\mathrm{x}$ time effect: $\mathrm{F}_{(8,372)}=2.468, \mathrm{p}<0.05$ ).

\section{Amphetamine self-administration in $\mathrm{SERT}^{-/-}$and $\mathrm{SERT}^{+/+}$rats.}

As expected, the escalation of AMPH intake over the daily self-administration sessions was higher in $\operatorname{LgA}$ than ShA rats (Fig 3A vs Fig 3B: access x self-administration session effect, $\mathrm{F}_{(3,73)}=3.458, \mathrm{p}=0.023$ ), and influenced by SERT genotype (Fig 3A and Fig 3B: genotype $\mathrm{x}$ access $\mathrm{x}$ self-administration session effect: $\left.\mathrm{F}_{(3,172)}=3.172, \mathrm{p}=0.021\right)$. More specifically, $\mathrm{SERT}^{-/}$rats were found to increase the daily intake of AMPH under LgA (Fig 3B: genotype $x$ self-administration session effect: $F_{(3,83)}=3.892, p=0.010$ ), but not under ShA conditions (Fig 3A: genotype x self-administration session effect: not significant: n.s.). No differences between $\mathrm{SERT}^{-/-}$and SERT ${ }^{+/+}$rats were found for the number of incorrect lever presses (supplementary Fig 1A and supplementary Fig 1B: genotype (x self-administration session) effect: n.s.) and timeout responses (supplementary Fig 1C and supplementary Fig 1D: genotype (x self-administration session) effect: n.s.).

Effect of ShA and $\operatorname{LgA}$ amphetamine self-administration on the glutamate presynaptic terminal in $\mathrm{SERT}^{+/+}$and $\mathrm{SERT}^{-/-}$rats .

We first focused our attention on the presynaptic terminal by analyzing the protein expression of the vesicular glutamate transporter (vGluT1) that is responsible of glutamate storage in presynaptic vesicles and it is considered an indirect index of glutamate release from the presynaptic neuron to the extracellular space (El Mestikawy et al., 2011). In the cNAc, two-way ANOVA revealed a main effect of AMPH access $\left(\mathrm{F}_{(2,57)}=3.46\right.$, $\mathrm{p}=0.038)$, genotype $\left(\mathrm{F}_{(1,57)}=25.57, \mathrm{p}<0.0001\right)$ and an AMPH access $\mathrm{x}$ genotype interaction $\left(\mathrm{F}_{(2,57)}=18.20\right.$, $\mathrm{p}<0.0001$; Fig 4a). Further intergroup sub-testing revealed increased expression of vGluT1 in $\mathrm{SERT}^{-/-}$rats exposed to either ShA (+58\% vs SERT ${ }^{-/-}$-naive, $\left.\mathrm{p}<0.0001\right)$ or $\operatorname{LgA}\left(+46 \% \mathrm{vs} \mathrm{SERT}^{-/-}\right.$-naive, $\left.\mathrm{p}=0.0003\right)$ with no effects in $\mathrm{SERT}^{+/+}$rats following the same procedures.

In the sNAc, two-way ANOVA revealed a main effect of AMPH access $\left(\mathrm{F}_{(2,55)}=311.50, \mathrm{p}<0.0001\right)$, genotype $\left(\mathrm{F}_{(1,55)}=83.97, \mathrm{p}<0.0001\right)$ and an AMPH access $\mathrm{x}$ genotype interaction $\left(\mathrm{F}_{(2,55)}=7.425, \mathrm{p}=0.0014 ; \mathrm{Fig} 4 \mathrm{~b}\right)$. In this sub region of the NAc, vGluT1 expression was reduced in SERT ${ }^{-/-}$-naive rats $\left(-41 \%\right.$ vs SERT ${ }^{+/+}$-naive, $\mathrm{p}=0.0471$ ). The lack of SERT influenced the response to the different regimens of AMPH exposure. In fact, while vGluT1 protein levels in SERT ${ }^{-/-}$were increased following ShA (+36\% vs SERT ${ }^{-/}-$-naive, $\left.\mathrm{p}=0.0396\right)$ but not $\mathrm{LgA}$ to $\mathrm{AMPH}$, in $\mathrm{SERT}^{+/+}$rats, the expression of vGluT1 was increased independently from the ShA $\left(+41 \%\right.$ vs SERT ${ }^{+/+}$-naive, $\left.\mathrm{p}=0.0145\right)$ or $\operatorname{LgA}\left(+61 \%\right.$ vs SERT ${ }^{+/+}$-naive, $\left.\mathrm{p}<0.0001\right)$ procedure.

Effect of ShA and LgA amphetamine self-administration on glial cells in SERT ${ }^{+/+}$and SERT $^{-/-}$ rats.

We next investigated the protein expression of the glial glutamate transporter (GLT-1) that is responsible of the reuptake of glutamate back into the glial cells from the extracellular space (Roberts-Wolfe \& Kalivas, 2015). In the cNAc two-way ANOVA revealed a significant AMPH access effect $\left(\mathrm{F}_{(2,57)}=10.94\right.$, $\mathrm{p}<0.0001)$ and an effect of genotype $\left(\mathrm{F}_{(1,57)}=9.349, \mathrm{p}=0.0034\right)$, whereas in the sNAc an effect of AMPH access $\left(\mathrm{F}_{(2,56)}=5.055, \mathrm{p}=0.0096\right)$, but no genotype effect was found. In both cNAc and sNAc, two-way ANOVA revealed a significant AMPH access $\mathrm{x}$ genotype interaction $\left(\mathrm{cNAc}: \mathrm{F}_{(2,57)}=3.444, \mathrm{p}=0.0387\right.$, Fig 5a; sNAc: $\mathrm{F}_{(2,56)}=3.347, \mathrm{p}=0.0423$, Fig $\left.5 \mathrm{~b}\right)$. Examining the individual treatment effects, we found that SERT deletion in both cNAc and sNAc led to a significant increase of GLT-1 expression in response to the 
LgA (cNAc: $+29 \%$ vs SERT ${ }^{-/-}$-naive, $\mathrm{p}=0.0115 ;+27 \%$ vs SERT ${ }^{-/-}$-ShA, $\mathrm{p}=0.002 ;$ sNAc: $^{2} 29 \%$ vs SERT $^{-/-}$ naive, $\mathrm{p}=0.0184 ;+25 \%$ vs SERT ${ }^{-/-}$ShA, $\left.\mathrm{p}=0.009\right)$, but not to the ShA procedure, whereas no significant effect was observed in $\mathrm{SERT}^{+/+}$rats.

Effect of ShA and $\operatorname{LgA}$ amphetamine self-administration on NMDA receptor subunits and the respective scaffolding proteins in the post-synaptic terminal of $\mathrm{SERT}^{+/+}$and $\mathrm{SERT}^{-/-}$rats .

The next step was to investigate the protein expression of the main subunits of the NMDA receptors. In the cNAc, two-way ANOVA of GluN1 levels, the obligatory subunit of NMDA receptors revealed an effect of genotype $\left(\mathrm{F}_{(1,57)}=4.544, \mathrm{p}=0.0374\right)$ and a significant $\mathrm{AMPH}$ access $\mathrm{x}$ genotype interaction $\left(\mathrm{F}_{(2,57)}=8.103\right.$, $\mathrm{p}=0.0008$; Fig 6a). Post-hoc testing revealed that the expression of this subunit of the NMDA receptor is significantly increased following the $\mathrm{LgA}\left(+40 \% \mathrm{vs} \mathrm{SERT}^{-/-}\right.$-naive, $\left.\mathrm{p}=0.0056\right)$, but not the ShA procedure in SERT $^{-/-}$rats. Neither ShA, nor LgA, affected GluN1 expression in the cNAc of in SERT ${ }^{+/+}$rats. A different situation was, instead, observed in the sNAc. Two-way ANOVA revealed a main effect of AMPH access $\left(\mathrm{F}_{(2,53)}=3.314, \mathrm{p}=0.044\right)$, genotype $\left(\mathrm{F}_{(1,53)}=20.53, \mathrm{p}<0.0001\right)$ and a significant AMPH access $\mathrm{x}$ genotype interaction $\left(\mathrm{F}_{(2,53)}=4.675, \mathrm{p}=0.0135\right.$; Fig $\left.6 \mathrm{~b}\right)$. Upon sub-testing we found an increased expression of GluN1 in $\mathrm{SERT}^{-/-}$naive-rats $\left(+44 \%\right.$ vs $\mathrm{SERT}^{+/+}$-naive, $\left.\mathrm{p}=0.0006\right)$, which was not further enhanced by the ShA and the $\operatorname{LgA}$ procedure. Conversely, both the ShA $\left(+31 \%\right.$ vs SERT ${ }^{-/-}$-naive, $\left.\mathrm{p}=0.0085\right)$ and $\operatorname{LgA}(+32 \%$ vs SERT $^{-/-}$-naive, $\left.\mathrm{p}=0.0089\right)$ conditions caused an enhancement in GluN1 protein levels in $\mathrm{SERT}^{+/+}$rats.

We then investigated the expression of the accessory subunits of the NMDA receptor, i.e. GluN2A and GluN2B. In the cNAc, two-way ANOVA of GluN2A levels revealed a main effect of AMPH access $\left(\mathrm{F}_{(2,57)}=5.137\right.$, $\mathrm{p}=0.0089)$, and an AMPH access $\mathrm{x}$ genotype interaction effect $\left(\mathrm{F}_{(2,57)}=11.36, \mathrm{p}<0.0001\right.$; Fig $\left.6 \mathrm{c}\right)$; in the same subregion, for the GluN2B subunit, two-way ANOVA revealed a main effect of genotype $\left(\mathrm{F}_{(1,56)}=12.21\right.$, $\mathrm{p}=0.0009)$ and an AMPH access $\mathrm{x}$ genotype interaction $\left(\mathrm{F}_{(2,56)}=10.41, \mathrm{p}=0.0001\right.$; Fig 6e). GluN2A subunit expression was reduced in $\mathrm{SERT}^{-/}$naive-rats $\left(-32 \% \mathrm{vs} \mathrm{SERT}^{+/+}\right.$-naive, $\left.\mathrm{p}=0.0433\right)$; further, the post-hoc intergroup comparisons revealed that the removal of SERT caused a different response to both the ShA and $\mathrm{LgA}$ conditions. In fact, GluN2A protein levels were reduced following both ShA (-36\% vs SERT ${ }^{+/+}$ naive, $\mathrm{p}=0.0032)$ and $\operatorname{LgA}\left(-34 \%\right.$ vs SERT ${ }^{+/+}$-naive, $\left.\mathrm{p}=0.0063\right) \mathrm{AMPH}$ self-administration in $\mathrm{SERT}^{+/+}$rats, whereas in $\mathrm{SERT}^{-/}$rats no changes were found for ShA while GluN2A protein levels were up-regulated following $\operatorname{LgA}\left(+28 \%\right.$ vs SERT ${ }^{-/-}$-naive, $\mathrm{p}=0.0426 ;+27 \%$ vs SERT $\left.^{-/-}-\mathrm{ShA}, \mathrm{p}=0.0085\right)$. GluN2B levels were not altered in AMPH-exposed SERT ${ }^{+/+}$rats (ShA: $-18 \%$ vs SERT ${ }^{+/+}$-naive, $\mathrm{p}=0.5623 ; \mathrm{LgA}:-27 \%$ vs $\mathrm{SERT}^{+/+}$naive, $\mathrm{p}=0.1727)$. In SERT ${ }^{-/}$rats similarly to GluN2A, we found that the $\mathrm{LgA}\left(+42 \% \mathrm{vs} \mathrm{SERT}^{-/-}\right.$-naive, $\mathrm{p}=0.0071 ;+33 \%$ vs $\left.\mathrm{SERT}^{-/}-\mathrm{ShA}, \mathrm{p}=0.010\right)$, but not the ShA procedure significantly up-regulated the expression of this subunit.

In the sNAc, two-way ANOVA revealed only a significant genotype effect $\left(\mathrm{F}_{(1,55)}=19.76, \mathrm{p}<0.0001\right.$; Fig $\left.6 \mathrm{~d}\right)$ for GluN2A, and a significant AMPH access effect $\left(\mathrm{F}_{(2,56)}=15.03, \mathrm{p}<0.0001\right.$; Fig $\left.6 \mathrm{f}\right)$ for GluN2B.

Following the analysis of the NMDA receptor subunits, we investigated the expression of the main scaffolding protein of NMDA receptors, SAP102 (Won et al., 2017). In the cNAc, two-way ANOVA revealed a main effect of AMPH access $\left(\mathrm{F}_{(2,56)}=9.318, \mathrm{p}=0.0003\right)$ and an AMPH access $\mathrm{x}$ genotype interaction $\left(\mathrm{F}_{(2,56)}=4.476\right.$, $\mathrm{p}=0.0157$; Fig 7a). Post-hoc testing of the main treatment effects showed that both ShA $\left(-20 \%\right.$ vs SERT ${ }^{+/+_{-}}$ naive, $\mathrm{p}=0.0033)$ and $\operatorname{LgA}\left(-27 \%\right.$ vs SERT ${ }^{+/+}$-naive, $\left.\mathrm{p}=0.0001\right) \mathrm{AMPH}$ self-administration resulted in reduced SAP102 expression in SERT ${ }^{+/+}$rats, whereas no changes were observed under both experimental conditions in SERT ${ }^{-/-}$rats. In the sNAc, no significant changes in SAP102 levels were observed (Fig 7b).

Effect of ShA and LgA amphetamine self-administration on AMPA receptor subunits and their respective scaffolding proteins in the post-synaptic terminal of $\mathrm{SERT}^{+/+}$and $\mathrm{SERT}^{-/-}$rats .

We then moved to the protein expression analysis of the main subunits of the AMPA receptor, i.e. GluA1 and GluA2. In the cNAc, for both GluA1 and GluA2 subunits, two-way ANOVA revealed a significant AMPH access $x$ genotype interaction (GluA1: $\mathrm{F}_{(2,57)}=16.03, \mathrm{p}<0.0001$, Fig 8a; GluA2: $\mathrm{F}_{(2,57)}=13.79, \mathrm{p}<0.0001$, Fig $8 \mathrm{c})$. For GluA2 two-way ANOVA revealed also a main effect of genotype $\left(\mathrm{F}_{(2,57)}=13.79, \mathrm{p}=0.0006\right)$. 
SERT knock out reduced GluA1 (-31\% vs SERT ${ }^{+/+}$-naive, $\left.\mathrm{p}=0.0477\right)$, but not GluA2, levels. The LgA condition reduced GluA1 levels in $\mathrm{SERT}^{+/+}\left(-37 \%\right.$ vs SERT ${ }^{+/+}$-naive, $\left.\mathrm{p}=0.0017\right)$ while increasing it in SERT $^{-/-}\left(+34 \%\right.$ vs SERT ${ }^{-/-}$-naive, $\left.\mathrm{p}=0.0048\right)$. GluA2 levels were reduced following both ShA (-26\% vs SERT ${ }^{+/+}$-naive, $\left.\mathrm{p}=0.0447\right)$ and $\operatorname{LgA}\left(-32 \%\right.$ vs $\mathrm{SERT}^{+/+}$-naive, $\left.\mathrm{p}=0.0083\right) \mathrm{AMPH}$ self-administration in $\mathrm{SERT}^{+/+}$, while deletion of SERT increased GluA2 levels following both ShA (+27\% vs $\mathrm{SERT}^{-/-}$-naive, $\mathrm{p}=0.0329)$ and $\operatorname{LgA}\left(+32 \%\right.$ vs SERT ${ }^{-/}$-naive, $\left.\mathrm{p}=0.0071\right) \mathrm{AMPH}$ self-administration. In the sNAc, twoway ANOVA of both GluA1 and GluA2 AMPA subunits revealed a main effect of AMPH access (GluA1: $\mathrm{F}_{(2,57)}=17.17, \mathrm{p}<0.0001 ;$ GluA2: $\left.\mathrm{F}_{(2,58)}=4.995, \mathrm{p}=0.010\right)$, genotype (GluA1: $\mathrm{F}_{(1,57)}=17.81, \mathrm{p}<0.0001$; GluA2: $\left.\mathrm{F}_{(1,58)}=15.09, \mathrm{p}=0.0003\right)$ and an AMPH access $\mathrm{x}$ genotype interaction effect (GluA1: $\mathrm{F}_{(2,57)}=3.571$, $\mathrm{p}=0.0346$, Fig 8b; GluA2: $\mathrm{F}_{(2,58)}=3.301, \mathrm{p}=0.0439$, Fig 8d). Both ShA and LgA conditions resulted in an elevation in GluA1 and GluA2 protein levels in SERT ${ }^{+/+}$rats $\left(G l u A 1:\right.$ ShA $+34 \%$ vs SERT ${ }^{+/+}$-naive, $\mathrm{p}=0.0329 ; \operatorname{LgA}+37 \%$ vs SERT ${ }^{+/+}$-naive, $\mathrm{p}=0.0185 ;$ GluA2: ShA $+46 \%$ vs SERT ${ }^{+/+}$-naive, $\mathrm{p}=0.0259$; $\mathrm{LgA}+44 \%$ vs SERT ${ }^{+/+}$-naive, $\left.\mathrm{p}=0.0411\right)$ whereas, in $\mathrm{SERT}^{-/-}$rats, no changes were observed with the exception of the increased expression of GluA1 following $\operatorname{LgA}\left(+54 \% \mathrm{vs} \mathrm{SERT}^{-/}\right.$-naive, $\mathrm{p}=0.0001 ;-37 \%$ vs $\mathrm{SERT}^{-/-}$-ShA, $\left.\mathrm{p}=0.0017\right)$.

We then examined the expression of the scaffolding proteins specific for AMPA receptor subunits GluA1 and GluA2, i.e. SAP97 and GRIP, respectively (Won et al., 2017). In the cNAc, for both SAP97 and GRIP, two-way ANOVA revealed a main effect of AMPH access (SAP97: $\mathrm{F}_{(2,57)}=4.464, \mathrm{p}=0.0158$; GRIP: $\mathrm{F}_{(1,58)}=5.822, \mathrm{p}=0.0050$ ), and an AMPH access $\mathrm{x}$ genotype interaction (SAP97: $\mathrm{F}_{(2,57)}=5.152, \mathrm{p}=0.0088$, Fig 9a; GRIP: $\mathrm{F}_{(2,58)}=4.385, \mathrm{p}=0.0169$, Fig 9c). In $\mathrm{SERT}^{+/+}$rats, we found a significant reduction of SAP97 under LgA AMPH self-administration conditions only ( $-30 \%$ vs $\mathrm{SERT}^{+/+}$-naive, $\left.\mathrm{p}=0.0011\right)$, whereas GRIP levels were reduced under both ShA (-27\% vs SERT ${ }^{+/+}$-naive, $\left.\mathrm{p}=0.0108\right)$ and $\mathrm{LgA}$ conditions $(-32 \%$ vs SERT $^{+/+}$-naive, $\left.\mathrm{p}=0.0019\right)$. Conversely, under both experimental conditions, no significant changes were shown in $\mathrm{SERT}^{-/}$rats. In the sNAc, two-way ANOVA of both SAP97 and GRIP levels revealed a main effect of AMPH access (SAP97: $\mathrm{F}_{(2,57)}=7.647, \mathrm{p}=0.0011$; GRIP: $\mathrm{F}_{(1,57)}=3.795, \mathrm{p}=0.0284$ ) and an AMPH access $x$ genotype interaction $\left(\mathrm{SAP} 97: \mathrm{F}_{(2,57)}=15.86\right.$, p $<0.0001$, Fig 9b; GRIP: $\mathrm{F}_{(2,57)}=7.230, \mathrm{p}=0.0016$, Fig 9d). Post-hoc testing of the main treatment effects in $\mathrm{SERT}^{+/+}$rats revealed reduced expression of SAP97 following $\operatorname{LgA}\left(-25 \%\right.$ vs SERT ${ }^{+/+}$-naive, $\left.\mathrm{p}=0.0429\right)$, but not ShA, procedures whereas GRIP was similarly reduced by both experimental conditions (ShA: $-23 \%$ vs SERT ${ }^{+/+}$-naive, $\mathrm{p}=0.0150$; LgA: $-31 \%$ vs $\mathrm{SERT}^{+/+}$naive, $\mathrm{p}=0.0005)$. Conversely, in $\mathrm{SERT}^{-/-}$rats, we found an increase in the expression of SAP97 following the LgA (-30\% vs SERT ${ }^{-/}$-naive, $\mathrm{p}=0.0093 ;-41 \%$ vs SERT $\left.{ }^{-/}-\mathrm{ShA}, \mathrm{p}<0.0001\right)$, but not the ShA condition, whereas GRIP expression was decreased in $\mathrm{SERT}^{-/-}$naive-rats $\left(-29 \% \mathrm{vs} \mathrm{SERT}^{+/+}\right.$-naive, $\left.\mathrm{p}=0.0059\right)$ without further changes following both ShA and LgA AMPH self-administration.

\section{Discussion}

We found that $\mathrm{SERT}^{-/-}$rats displayed increased AMPH intake under LgA, but not ShA conditions. Furthermore, the psychomotor response to AMPH was significantly higher in SERT ${ }^{-/}$rats. Finally, we found that, after 24 hours of withdrawal from AMPH self-administration, the glutamatergic synapse was affected differently in $\mathrm{SERT}^{-/-}$versus $\mathrm{SERT}^{+/+}$rats, in a way that heavily relies on the subregion of the NAc (shell or core) and the type of AMPH access (ShA or LgA).

\section{Amphetamine's psychomotor and reinforcing effects}

Because of the pronounced genotype differences in the psychomotor effects of AMPH, one could argue that locomotor activity had an influence on the observed genotype differences after LgA to AMPH selfadministration. However, the lack of genotype differences in AMPH intake under ShA condition and in the number of incorrect responses suggests that the increased AMPH intake under LgA condition in SERT $\mathrm{ST}^{-/}$ versus $\mathrm{SERT}^{+/+}$rats did not result from increased AMPH-induced locomotor activity. Interestingly, while AMPH self-administration was only increased in SERT ${ }^{-/-}$rats under LgA but not ShA conditions, cocaine self-administration was increased in these animals under both conditions (Verheij et al., 2018). While we did not conduct a direct comparison study, this implies that the serotonin influence on AMPH and cocaine 
self-administration is different. A potential explanation is that AMPH and cocaine have a different affinity for SERT (Howell \& Kimmel, 2008), and that serotonin, next to dopamine, contributes to psychostimulant's reinforcing effects too.

\section{Amphetamine effects in Nucleus Accumbens shell (sNAc)}

Twenty-four hours into withdrawal from AMPH self-administration, we found that vGluT1 expression was reduced at baseline in the sNAc of $\mathrm{SERT}^{-/-}$rats, compared to $\mathrm{SERT}^{+/+}$rats, suggesting reduced vesicular release of glutamate in $\mathrm{SERT}^{-/-}$rats with consequently lower extracellular levels in the synapse. Of note, SERT $^{+/+}$rats showed increased vGluT1 expression after both ShA and LgA, whereas SERT ${ }^{-/-}$rats exhibited an increase of the vesicular transporter following ShA and no change following LgA. Since the extracellular levels of glutamate are determined by a balance between its release and reuptake, the evidence that the accumbal expression of the plasmalemmal glutamate transporter GLT-1 is not altered by AMPH in SERT ${ }^{+/+}$ rats might be indicative of an increased AMPH-induced overflow of glutamate in the sNAc of these animals. Instead, GLT-1 expression is increased in $\mathrm{SERT}^{-/-} \mathrm{LgA}$ rats, possibly reducing the extracellular levels of glutamate, whereas it is not changed following ShA. This suggests that SERT deletion may be associated with an increased glutamate overflow as a consequence of a ShA-induced increase of vGluT1. On the other hand, SERT deletion may reduce glutamate overflow as a consequence of a LgA-induced increase of GLT-1 expression. Since the sNAc is critical for the reinforcing properties of psychostimulants (Guillem et al., 2014), the possibility exists that altered extracellular glutamate levels in this brain subregion of $\mathrm{SERT}^{-/-}$rats may affect drug seeking.

The analysis of the expression of glutamate receptors in the sNAc revealed differences between NMDA and AMPA receptors. At baseline, a significant change was represented by the increased expression of GluN1 in $\mathrm{SERT}^{-/-}$rats, perhaps resulting from the reduced glutamate release caused by the decreased expression of vGluT1. Following both ShA and $\operatorname{LgA}$ AMPH self-administration, we found increased expression of GluN1 in SERT ${ }^{+/+}$rats, suggesting that the proposed higher release of glutamate in SERT S $^{+/+}$rats is not compensated by a significant reduction in the main NMDA subunit. Conversely, in $\mathrm{SERT}^{-/}$rats, neither ShA nor LgA are able to further enhance the expression of GluN1, suggesting that SERT deletion-induced changes in GluN1 expression may be maximal. Notably, the observed increase in GluN1 expression were not accompanied by an increase in the levels of the accessory subunits GluN2A and GluN2B and of its anchoring protein SAP102, suggesting receptor instability following both ShA and LgA.

Regarding AMPA receptors, we found no significant changes at baseline between the two genotypes. GluA1 and GluA2 expression was up-regulated following either ShA or LgA in SERT ${ }^{+/+}$rats. However, when analyzing the main scaffolding proteins for these two AMPA subunits, we found reduced expression of both SAP97 and GRIP in SERT ${ }^{+/+}$rats. These findings suggest that GluA1 and GluA2 receptors lose their stability following both drug regimens in SERT ${ }^{+/+}$rats. In SERT ${ }^{-/-}$rats, both GluA1 and SAP97 expression was significantly increased following LgA drug exposure, suggesting that LgA to AMPH increased the stability of at least the GluA1 receptor in these animals.

In $\mathrm{SERT}^{+/+}$rats, the proposed AMPH-induced increased release of glutamate, not compensated by changes in the expression of GLT-1, leads to an increased expression of the main glutamate receptors. Instead, the localization and, presumably, the functionality of these receptors is attenuated by the uncoupling of their respective auxiliary proteins independently from the duration of the AMPH exposure. Conversely, in SERT $/$ rats, the proposed reduced glutamate release is accompanied by enhanced reuptake only following LgA drug exposure, a combination that leads to a functional compensation only for GluA1 but not for GluA2 AMPA subunit nor for the different NMDA receptor subunits. Of note, the increased expression of GluA1, but not GluA2, AMPA subunit following LgA may lead to the formation of GluA2-lacking and $\mathrm{Ca}^{2+}$-permeable AMPARs, a mechanism known to drive addiction (Wolf, 2016).

\section{Amphetamine effects in Nucleus Accumbens core (cNAc)}

A different situation was observed in the cNAc. In fact, neither expression of protein regulating release, nor expression of protein regulating reuptake of glutamate were significantly changed at baseline in SERT'/- 
rats compared to their wild-type counterpart. In addition, no significant changes in these proteins were observed in the cNAc of SERT ${ }^{+/+}$rats exposed to either regimen of AMPH. Conversely, both ShA and LgA may have promoted a significant higher release of glutamate that was accompanied by a higher uptake in the LgA-, but not ShA, exposed in SERT-/-rats. The up-regulation of GLT-1 may represent an adaptive mechanism to buffer the increased release of glutamate, which is absent in ShA-exposed rats. This may indicate that changes in GLT-1 expression reflect compensation in the core, and synergism in the shell (see above). Interestingly, in the cNAc of SERT ${ }^{+/+}$rats, we noted that both ShA and LgA to AMPH reduced the expression of GluN2 and GluA2 receptor subunits, an effect observed also for their respective scaffolding proteins, i.e. SAP102, SAP97 and GRIP. Conversely, in SERT ${ }^{-/}$rats, LgA, but not ShA, significantly upregulated the three GluN and the two GluA subunits (with the exception of GluA2 in ShA-exposed rats), an effect that was not accompanied, though, by a parallel enhancement of the expression of their respective scaffolding proteins, pointing towards an AMPH-induced reduction in the stability of glutamatergic synapse of the cNAc of $\mathrm{SERT}^{-/-}$rats.

\section{Conclusion}

In conclusion, our data show that SERT removal and AMPH self-administration converge on a common set of glutamate abnormalities that may contribute to shape both the behavioral and molecular effects herein observed. The finding that, after ShA to AMPH, genotype differences at the level of the glutamate system are not accompanied by genotype differences in drug intake, suggest that the observed SERT-induced changes in the glutamate system functioning result from AMPH self-administration. On the other hand, the finding that, after $\operatorname{LgA}$ to AMPH, genotype differences at the level of the glutamate system are accompanied by genotype differences in drug intake, suggests that SERT-induced changes in the glutamate system may also drive AMPH self-administration. It appears that the combination of SERT deletion and AMPH exposure leads to effects that are unique and distinguishable from those of the individual experimental conditions. Accordingly, we can make several considerations: 1) the glutamate system is differently regulated by AMPH in $\mathrm{SERT}^{-/-}$rats when compared to $\mathrm{SERT}^{+/+}$rats; this is observed not only in terms of expression of the different glutamate receptors, but also in terms of their retention (i.e. stability) at synaptic sites, 2) the molecular determinants of the glutamate synapse are differently regulated in cNAc and sNAc confirming that these two subregions have their own function in regulating drug dependence and, 3) the LgA paradigm shapes the glutamatergic synapse differently from the ShA condition. Taken together, the perturbation of serotonin activity may, via dysregulation of glutamate homeostasis in these NAc regions, contribute to drive the transition from goal-directed drug intake toward addictive states. Hence, we propose that manipulation of serotonergic and/or glutamatergic systems may affect not only cocaine, but also AMPH, dependence.

\section{Figure legends}

Figure 1. Schematic representation of the experimental paradigm performed in SERT $\mathrm{ST}^{-/}$and $\mathrm{SERT}^{+/+}$rats. (A) The psychomotor effects of AMPH were assessed immediately after AMPH injection and evaluated for 120 min. (B) AMPH self-administration training was performed during 10 days, after which the animals were subdivided into Short $(\mathrm{ShA})$ or Long $(\operatorname{LgA})$ access groups. During the next 18 days the rats were allowed to self-administer AMPH under either of these conditions. (C) $24 \mathrm{hrs}$ after the final ShA or LgA session rats were decapitated, and punches from the nucleus accumbens core (cNAc) and shell (sNAc) sub regions (localized using the Paxinos and Watson 2007 edition of the rat brain atlas) were collected for gene expression analyses of the glutamatergic synapse.

Figure 2. Psychomotor effects of AMPH in SERT ${ }^{+/+}$and SERT ${ }^{-/-}$animals. The AMPH-induced and dose-dependent increase in locomotor activity was stronger in $\mathrm{SERT}^{-/}$vs $\mathrm{SERT}^{+/+}$rats. Data represent mean +- SEM distance moved. ${ }^{*} \mathrm{p}<0.05$ versus respective $\mathrm{SERT}^{+/+}$vehicle; $\% \mathrm{p}<0.05$ versus respective $\mathrm{SERT}^{-/-}$vehicle; \# $\mathrm{p}<0.05$ versus amphetamine in $\mathrm{SERT}^{-/-}$rats; filled symbols versus baseline (time 0 ).

Figure 3. AMPH self-administration in $\mathrm{SERT}^{+/+}$and $\mathrm{SERT}^{-/-}$animals. The escalation of AMPH intake over the self-administration sessions was larger in rats under LgA than under ShA conditions (A vs B). The daily AMPH intake was larger in $\mathrm{SERT}^{-/-}$versus $\mathrm{SERT}^{+/+}$rats under $\mathrm{LgA}(\mathrm{B})$, but not ShA (A) conditions. 
Data are represented as mean +- SEM. n.s.: not significant. * p[?]0.05 (one-sided t-test) versus $\mathrm{SERT}^{+/+}$.

Figure 4 . Interaction between SERT deletion and AMPH self-administration on the vesicular glutamate transporter 1 (vGluT1) in the cNAc and sNAc. Protein levels of vGluT1 in (A) cNAc and (B) sNAc are expressed as percentages of SERT ${ }^{+/+}$-naive rats. Below the graphs, representative immunoblots are shown for vGluT1 $(60 \mathrm{kDa})$ and $\beta$-actin $(43 \mathrm{kDa})$ proteins in the homogenate of cNAc and sNAc. Histograms represent the mean \pm SEM of the following number of rats: naïve $\left(\mathrm{SERT}^{+/+} \mathrm{n}=6\right.$; $\mathrm{SERT}^{-/-} \mathrm{n}=6$ ) ShA $\left(\mathrm{SERT}^{+/+} \mathrm{n}=14 ; \mathrm{SERT}^{-/-} \mathrm{n}=13\right)$ and $\operatorname{LgA}\left(\mathrm{SERT}^{+/+} \mathrm{n}=12 ; \mathrm{SERT}^{-/-} \mathrm{n}=12\right) .{ }^{*} \mathrm{p}<0.05,{ }^{* * *} \mathrm{p}<0.001$ versus SERT $^{+/+}$-naive; ${ }^{\#} \mathrm{p}<0.05,{ }^{\# \# \#} \mathrm{p}<0.001$ versus $\mathrm{SERT}^{-/-}$-naive (Tukey's multiple comparisons test).

$\mathrm{N}=$ naïve, $\mathrm{S}=$ Amphetamine Short-Access; $\mathrm{L}=$ Amphetamine Long-Access

Figure 5 . Interaction between SERT deletion and AMPH self-administration on the glial glutamate transporter 1 (GLT-1) in the cNAc and sNAc. Protein levels of GLT-1 in (A) cNAc and (B) sNAc are expressed as percentages of SERT ${ }^{+/+}$-naive rats. Below the graphs, representative immunoblots are shown for GLT-1 $(62 \mathrm{kDa})$ and $\beta$-actin $(43 \mathrm{kDa})$ proteins in the homogenate of cNAc and sNAc. Histograms represent the

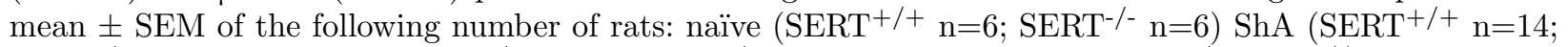
$\left.\mathrm{SERT}^{-/-} \mathrm{n}=13\right)$ and LgA $\left(\mathrm{SERT}^{+/+} \mathrm{n}=12 ; \mathrm{SERT}^{-/-} \mathrm{n}=12\right) .{ }^{\#} \mathrm{p}<0.05$ versus $\mathrm{SERT}^{-/-}$-naïve; ${ }^{\$ \$} \mathrm{p}<0.01$ versus SERT $^{-/-}$-ShA (Tukey's multiple comparisons test).

$\mathrm{N}=$ naïve, $\mathrm{S}=$ Amphetamine Short-Access; $\mathrm{L}=$ Amphetamine Long-Access

Figure 6 . Interaction between SERT deletion and AMPH self-administration on the NMDA receptor subunits in the cNAc and sNAc. Data show GluN1 levels in (A) cNAc and (B) sNAc, GluN2A levels in (C) cNAc and (D) sNAc, and GluN2B levels in (E) cNAc and (F) sNAc. In the lower panel (G) representative immunoblots are shown for GluN2A (180 kDa), GluN2B (180 kDa), GluN1 (120 kDa) and $\beta$-actin (43 kDa) proteins in the cNAc and sNAc homogenates of SERT ${ }^{+/+}$and $\mathrm{SERT}^{-/-}$rats exposed to AMPH. Results are expressed as percentages of SERT ${ }^{+/+}$-naive rats. Histograms represent the mean \pm SEM of the following number of rats: naïve $\left(\mathrm{SERT}^{+/+} \mathrm{n}=6 ; \mathrm{SERT}^{-/-} \mathrm{n}=6\right) \mathrm{ShA}\left(\mathrm{SERT}^{+/+} \mathrm{n}=14 ; \mathrm{SERT}^{-/-} \mathrm{n}=13\right)$ and $\mathrm{LgA}$ $\left(\mathrm{SERT}^{+/+} \mathrm{n}=12 ; \mathrm{SERT}^{-/-} \mathrm{n}=12\right) .{ }^{*} \mathrm{p}<0.05,{ }^{* *} \mathrm{p}<0.01,{ }^{* * *} \mathrm{p}<0.001$ versus $\mathrm{SERT}^{+/+}$-nä̈ve; ${ }^{\#} \mathrm{p}<0.05$, \#\# $\mathrm{p}<0.01$ versus $\mathrm{SERT}^{-/-}$-naïve; ${ }^{\$} \mathrm{p}<0.05,{ }^{\$ \$} \mathrm{p}<0.01$ versus $\mathrm{SERT}^{-/-}$-ShA (Tukey's multiple comparisons test).

$\mathrm{N}=$ naïve, $\mathrm{S}=$ Amphetamine Short-Access; $\mathrm{L}=$ Amphetamine Long-Access

Figure 7 . Interaction between SERT deletion and AMPH self-administration on the scaffolding protein SAP102 in the cNAc and sNAc. Data show protein levels of SAP102 in the (A) cNAc and (B) sNAc expressed as percentage of SERT ${ }^{+/+}$-naive rats. Below the graphs, representative immunoblots are shown for SAP102 $(102 \mathrm{kDa})$ and $\beta$-actin $(43 \mathrm{kDa})$ proteins in the homogenate of cNAc and sNAc. Histograms represent the mean \pm SEM of the following number of rats: naïve (SERT ${ }^{+/+} n=6$; SERT $\left.^{-/-} n=6\right)$ ShA $\left(\mathrm{SERT}^{+/+}\right.$ $\left.\mathrm{n}=14 ; \mathrm{SERT}^{-/-} \mathrm{n}=13\right)$ and LgA $\left(\mathrm{SERT}^{+/+} \mathrm{n}=12 ; \mathrm{SERT}^{-/-} \mathrm{n}=12\right) .{ }^{* *} \mathrm{p}<0.01,{ }^{* * *} \mathrm{p}<0.001$ versus $\mathrm{SERT}^{+/+_{-}}$ naïve (Tukey's multiple comparisons test).

$\mathrm{N}=$ naïve, $\mathrm{S}=$ Amphetamine Short-Access; L = Amphetamine Long-Access

Figure 8 . Interaction between SERT deletion and AMPH self-administration on the AMPA receptor subunits in the cNAc and sNAc. Data show GluA1 levels in the (A) cNAc and (B) sNAc, and GluA2 levels in the $(\mathrm{C})$ cNAc and (D) sNAc. In the lower panel (E) representative immunoblots are shown for GluA1 (108 $\mathrm{kDa})$, GluA2 $(108 \mathrm{kDa})$ and $\beta$-actin $(43 \mathrm{kDa})$ proteins in the cNAc and sNAc homogenates of SERT ${ }^{+/+}$ and $\mathrm{SERT}^{-/}$rats exposed to AMPH. Results are expressed as percentages of SERT ${ }^{+/+}$-naive rats. Histograms represent the mean $\pm \mathrm{SEM}$ of the following number of rats: naïve $\left(\mathrm{SERT}^{+/+} \mathrm{n}=6\right.$; $\mathrm{SERT}^{-/-} \mathrm{n}=6$ ) ShA $\left(\mathrm{SERT}^{+/+} \mathrm{n}=14 ; \mathrm{SERT}^{-/-} \mathrm{n}=13\right)$ and $\operatorname{LgA}\left(\mathrm{SERT}^{+/+} \mathrm{n}=12 ; \mathrm{SERT}^{-/-} \mathrm{n}=12\right) .{ }^{*} \mathrm{p}<0.05{ }^{* *} \mathrm{p}<0.01$ versus SERT ${ }^{+/+}$-naïve; ${ }^{\#} \mathrm{p}<0.05,{ }^{\# \#} \mathrm{p}<0.01,{ }^{\# \# \#} \mathrm{p}<0.001$ versus $\mathrm{SERT}^{-/-}$-naïve; ${ }^{\$} \mathrm{p}<0.01$ versus $\mathrm{SERT}^{-/-}$-ShA (Tukey's multiple comparisons test).

$\mathrm{N}=$ naïve, $\mathrm{S}=$ Amphetamine Short-Access; $\mathrm{L}=$ Amphetamine Long-Access 
Figure 9 . Interaction between SERT deletion and AMPH self-administration on the scaffolding proteins SAP97 and GRIP in the cNAc and sNAc. Data show SAP97 levels in the (A) cNAc and (B) sNAc, and GRIP levels in the (C) cNAc and (D) sNAc. In the lower panel (E) representative immunoblots are shown for GRIP $(120 \mathrm{kDa})$, SAP97 $(97 \mathrm{kDa})$ and $\beta$-actin $(43 \mathrm{kDa})$ proteins in the cNAc and sNAc homogenates of SERT ${ }^{+/+}$ and $\mathrm{SERT}^{-/}$rats exposed to AMPH. Results are expressed as percentage of $\mathrm{SERT}^{+/{ }^{+}}$-naive rats. Histograms represent the mean $\pm \mathrm{SEM}$ of the following number of rats: naïve $\left(\mathrm{SERT}^{+/+} \mathrm{n}=6\right.$; $\mathrm{SERT}^{-/-} \mathrm{n}=6$ ) ShA $\left(\mathrm{SERT}^{+/+} \mathrm{n}=14 ; \mathrm{SERT}^{-/-} \mathrm{n}=13\right)$ and LgA $\left(\mathrm{SERT}^{+/+} \mathrm{n}=12 ; \mathrm{SERT}^{-/-} \mathrm{n}=12\right) .{ }^{*} \mathrm{p}<0.05,{ }^{* *} \mathrm{p}<0.01,{ }^{* * *} \mathrm{p}<0.001$ versus $\mathrm{SERT}^{+/+}$-naïve; $\# \# \mathrm{p}<0.01$ versus $\mathrm{SERT}^{-/-}$-naïve; ${ }^{\$ \$ \$} \mathrm{p}<0.001$ versus $\mathrm{SERT}^{-/-}{ }^{-S h A}$ (Tukey's multiple comparisons test).

$\mathrm{N}=$ naïve, $\mathrm{S}=$ Amphetamine Short-Access; L= Amphetamine Long-Access

Figure 10. Summary of the protein expression findings in the nucleus accumbens shell and core of naïve $\mathrm{SERT}^{+/+}$and $\mathrm{SERT}^{-/-}$rats versus $\mathrm{SERT}^{+/+}$and $\mathrm{SERT}^{-/-}$rats exposed to ShA and LgA cocaine selfadministration.

$\mathrm{cNAC}=$ nucleus accumbens core, $\mathrm{sNAC}=$ nucleus accumbens shell, $\mathrm{ShA}=$ Amphetamine Short-Access; $\operatorname{LgA}=$ amphetamine Long-Access, vGlut1=vesicular glutamate transporter 1, GLT-1=glial glutamate transporter 1, NMDA=NMDA receptor unit (e.g. GluN1), Scaffolding=scaffolding protein (e.g. SAP102), AMPA=AMPA receptor subunit

Supplementary figure 1 . Number of incorrect lever presses (A, B) and timeout responses (C, D) during AMPH self-administration in $\mathrm{SERT}^{-/-}$and $\mathrm{SERT}^{+/+}$rats under ShA and LgA conditions. No genotype differences were observed. Data are represented as mean \pm SEM. n.s.: not significant.

Supplementary figure 2. Uncropped immunoblot related to the expression levels of GluN2A (180 kDa), GluN2B (180 kDa), GluN1 (120 kDa), GRIP (135 kDa), SAP97 (97 kDa), SAP102 (102 kDa), GluA1 (108 $\mathrm{kDa})$, GluA2 (108 kDa), vGlut1 (67 kDa), GLT-1 (62 kDa) and $\beta$-Actin (43 kDa) measured in the homogenate of the cNAc of SERT ${ }^{+/+}$and $\mathrm{SERT}^{-/-}$rats exposed to AMPH ShA and $\mathrm{LgA}$ protocol presented in Fig 4, Fig 5, Fig 6, Fig 7, Fig 8, Fig 9. Samples were loaded in three different gels as shown in panel A, B, C.

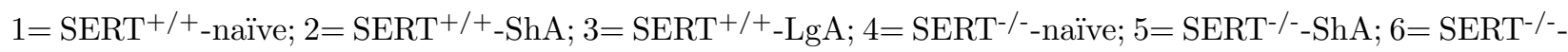
LgA.

Supplementary figure 3 . Uncropped immunoblot related to the expression levels of GluN2A (180 kDa), GluN2B (180 kDa), GluN1 (120 kDa), GRIP (135 kDa), SAP97 (97 kDa), SAP102 (102 kDa), GluA1 (108 $\mathrm{kDa})$, GluA2 (108 kDa), vGlut1 (67 kDa), GLT-1 (62 kDa) and $\beta$-Actin (43 kDa) measured in the homogenate of the sNAc of SERT ${ }^{+/+}$and $\mathrm{SERT}^{-/-}$rats exposed to AMPH ShA and $\operatorname{LgA}$ protocol presented in Fig 4, Fig 5, Fig 6, Fig 7, Fig 8, Fig 9. Samples were loaded in three different gels as shown in panel A, B, C.

\section{References}

Ahmed, S. H., \& Koob, G. F. (1998). Transition from moderate to excessive drug intake: change in hedonic set point. Science, 282 (5387), 298-300. doi:10.1126/science.282.5387.298

Caffino, L., Mottarlini, F., Van Reijmersdal, B., Telese, F., Verheij, M. M. M., Fumagalli, F., \& Homberg, J. R. (2020). The role of the serotonin transporter in prefrontal cortex glutamatergic signaling following shortand long-access cocaine self-administration.Addict Biol , e12896. doi:10.1111/adb.12896

Caffino, L., Verheij, M. M. M., Que, L., Guo, C., Homberg, J. R., \& Fumagalli, F. (2019). Increased cocaine self-administration in rats lacking the serotonin transporter: a role for glutamatergic signaling in the habenula. Addict Biol, 24 (6), 1167-1178. doi:10.1111/adb.12673

Caspi, A., Hariri, A. R., Holmes, A., Uher, R., \& Moffitt, T. E. (2010). Genetic sensitivity to the environment: the case of the serotonin transporter gene and its implications for studying complex diseases and traits. $A m$ J Psychiatry, 167 (5), 509-527. doi:10.1176/appi.ajp.2010.09101452

Curtis, M. J., Alexander, S., Cirino, G., Docherty, J. R., George, C. H., Giembycz, M. A., . . . Ahluwalia, A. (2018). Experimental design and analysis and their reporting II: updated and simplified guidance for 
authors and peer reviewers. Br J Pharmacol, 175 (7), 987-993. doi:10.1111/bph.14153

Degenhardt, L., Baxter, A. J., Lee, Y. Y., Hall, W., Sara, G. E., Johns, N., . . Vos, T. (2014). The global epidemiology and burden of psychostimulant dependence: findings from the Global Burden of Disease Study 2010. Drug Alcohol Depend, 137 , 36-47. doi:10.1016/j.drugalcdep.2013.12.025

El Mestikawy, S., Wallen-Mackenzie, A., Fortin, G. M., Descarries, L., \& Trudeau, L. E. (2011). From glutamate co-release to vesicular synergy: vesicular glutamate transporters. Nat Rev Neurosci, 12 (4), 204-216. doi:10.1038/nrn2969

Enoch, M. A., Gorodetsky, E., Hodgkinson, C., Roy, A., \& Goldman, D. (2011). Functional genetic variants that increase synaptic serotonin and 5-HT3 receptor sensitivity predict alcohol and drug dependence.Mol Psychiatry, 16 (11), 1139-1146. doi:10.1038/mp.2010.94

Everitt, B. J., \& Robbins, T. W. (2016). Drug Addiction: Updating Actions to Habits to Compulsions Ten Years On. Annu Rev Psychol, 67 , 23-50. doi:10.1146/annurev-psych-122414-033457

Faraone, S. V. (2018). The pharmacology of amphetamine and methylphenidate: Relevance to the neurobiology of attention-deficit/hyperactivity disorder and other psychiatric comorbidities. Neurosci Biobehav Rev, 87 , 255-270. doi:10.1016/j.neubiorev.2018.02.001

Gerra, G., Zaimovic, A., Garofano, L., Ciusa, F., Moi, G., Avanzini, P., . . . Donnini, C. (2007). Perceived parenting behavior in the childhood of cocaine users: relationship with genotype and personality traits. $A m$ $J$ Med Genet B Neuropsychiatr Genet, $144 B$ (1), 52-57. doi:10.1002/ajmg.b.30388

Giannotti, G., Caffino, L., Mottarlini, F., Racagni, G., \& Fumagalli, F. (2016). Region-specific effects of developmental exposure to cocaine on fibroblast growth factor-2 expression in the rat brain.Psychopharmacology (Berl), 233 (14), 2699-2704. doi:10.1007/s00213-016-4315-9

Gipson, C. D., \& Bardo, M. T. (2009). Extended access to amphetamine self-administration increases impulsive choice in a delay discounting task in rats. Psychopharmacology (Berl), 207 (3), 391-400. doi:10.1007/s00213009-1667-4

Guillem, K., Ahmed, S. H., \& Peoples, L. L. (2014). Escalation of cocaine intake and incubation of cocaine seeking are correlated with dissociable neuronal processes in different accumbens subregions.Biol Psychiatry, 76 (1), 31-39. doi:10.1016/j.biopsych.2013.08.032

Holmes, A. J., Bogdan, R., \& Pizzagalli, D. A. (2010). Serotonin transporter genotype and action monitoring dysfunction: a possible substrate underlying increased vulnerability to depression. Neuropsychopharmacology, 35 (5), 1186-1197. doi:10.1038/npp.2009.223

Homberg, J. R., De Boer, S. F., Raaso, H. S., Olivier, J. D., Verheul, M., Ronken, E., . . . Cuppen, E. (2008). Adaptations in pre- and postsynaptic 5-HT1A receptor function and cocaine supersensitivity in serotonin transporter knockout rats. Psychopharmacology (Berl), 200 (3), 367-380. doi:10.1007/s00213-008-1212-x

Homberg, J. R., Olivier, J. D., Smits, B. M., Mul, J. D., Mudde, J., Verheul, M., . . . Cuppen, E. (2007). Characterization of the serotonin transporter knockout rat: a selective change in the functioning of the serotonergic system. Neuroscience, 146 (4), 1662-1676. doi:10.1016/j.neuroscience.2007.03.030

Homberg, J. R., Wohr, M., \& Alenina, N. (2017). Comeback of the Rat in Biomedical Research. ACS Chem Neurosci, 8 (5), 900-903. doi:10.1021/acschemneuro.6b00415

Howell, L. L., \& Kimmel, H. L. (2008). Monoamine transporters and psychostimulant addiction. Biochem Pharmacol, 75 (1), 196-217. doi:10.1016/j.bcp.2007.08.003

Kalivas, P. W., \& Volkow, N. D. (2005). The neural basis of addiction: a pathology of motivation and choice. Am J Psychiatry, 162 (8), 1403-1413. doi:10.1176/appi.ajp.162.8.1403 
Kilkenny, C., Browne, W. J., Cuthill, I. C., Emerson, M., \& Altman, D. G. (2010). Improving bioscience research reporting: The ARRIVE guidelines for reporting animal research. J Pharmacol Pharmacother, 1 (2), 94-99. doi:10.4103/0976-500X.72351

Koob, G. F. (1992). Neural mechanisms of drug reinforcement. Ann N Y Acad Sci, 654, 171-191. doi:10.1111/j.1749-6632.1992.tb25966.x

Lile J, Nader M. (2003) The abuse liability and therapeutic potential of drugs evaluated

for cocaine addiction as predicted by animal models. Curr Neuropharmacol. 1(1), 21-46(26) doi:10.2174/1570159033360566

Mannelli, P., Patkar, A. A., Murray, H. W., Certa, K., Peindl, K., Mattila-Evenden, M., \& Berrettini, W. H. (2005). Polymorphism in the serotonin transporter gene and response to treatment in African American cocaine and alcohol-abusing individuals. Addict Biol, 10 (3), 261-268. doi:10.1080/13556210500235540

Martin-Santos, R., Torrens, M., Poudevida, S., Langohr, K., Cuyas, E., Pacifici, R., . . . de la Torre, R. (2010). 5-HTTLPR polymorphism, mood disorders and MDMA use in a 3-year follow-up study. Addict Biol, 15 (1), 15-22. doi:10.1111/j.1369-1600.2009.00180.x

Natarajan, R., \& Yamamoto, B. K. (2011). The Basal Ganglia as a Substrate for the Multiple Actions of Amphetamines. Basal Ganglia, 1 (2), 49-57. doi:10.1016/j.baga.2011.05.003

Oakly, A. C., Brox, B. W., Schenk, S., \& Ellenbroek, B. A. (2014). A genetic deletion of the serotonin transporter greatly enhances the reinforcing properties of MDMA in rats. Mol Psychiatry, 19 (5), 534-535. doi:10.1038/mp.2013.75

Oliva, C., Escobedo, P., Astorga, C., Molina, C., \& Sierralta, J. (2012). Role of the MAGUK protein family in synapse formation and function. Dev Neurobiol, 72 (1), 57-72. doi:10.1002/dneu.20949

Paxinos G, Watson C (2007) The rat brain in stereotaxic coordinates, 6th edn.

Academic Press/Elsevier, Amsterdam

Pettie, M., Oakly, A., Harper, D. N., \& Ellenbroek, B. A. (2019). A genetic deletion of the serotonin transporter differentially influences the behavioural effects of MDMA. J Psychopharmacol, 33 (3), 355-363. doi: $10.1177 / 0269881118822156$

Roberts-Wolfe, D. J., \& Kalivas, P. W. (2015). Glutamate Transporter GLT-1 as a Therapeutic Target for Substance Use Disorders. CNS Neurol Disord Drug Targets, 14 (6), 745-756. doi:10.2174/1871527314666150529144655

Rocha, B. A., Fumagalli, F., Gainetdinov, R. R., Jones, S. R., Ator, R., Giros, B., . . . Caron, M. G. (1998). Cocaine self-administration in dopamine-transporter knockout mice. Nat Neurosci, 1 (2), 132-137. doi: $10.1038 / 381$

Rothman, R. B., \& Glowa, J. R. (1995). A review of the effects of dopaminergic agents on humans, animals, and drug-seeking behavior, and its implications for medication development. Focus on GBR 12909.Mol Neurobiol, 11 (1-3), 1-19. doi:10.1007/BF02740680

Schipper, P., Hiemstra, M., Bosch, K., Nieuwenhuis, D., Adinolfi, A., Glotzbach, S., . . . Homberg, J. R. (2019). The association between serotonin transporter availability and the neural correlates of fear bradycardia. Proc Natl Acad Sci U S A, 116 (51), 25941-25947. doi:10.1073/pnas.1904843116

Shaffer, C., Guo, M. L., Fibuch, E. E., Mao, L. M., \& Wang, J. Q. (2010). Regulation of group I metabotropic glutamate receptor expression in the rat striatum and prefrontal cortex in response to amphetamine in vivo. Brain Res, 1326 , 184-192. doi:10.1016/j.brainres.2010.02.062

Smits, B. M., Mudde, J. B., van de Belt, J., Verheul, M., Olivier, J., Homberg, J., . . . Cuppen, E. (2006). Generation of gene knockouts and mutant models in the laboratory rat by ENU-driven targetselected mutagenesis. Pharmacogenet Genomics, 16 (3), 159-169. doi:10.1097/01.fpc.0000184960.82903.8f 
Traynelis, S. F., Wollmuth, L. P., McBain, C. J., Menniti, F. S., Vance, K. M., Ogden, K. K., . . . Dingledine, R. (2010). Glutamate receptor ion channels: structure, regulation, and function. Pharmacol Rev, 62 (3), 405-496. doi:10.1124/pr.109.002451

UNODOC World Drug Report (2018) https://www.unodc.org/wdr2018/

Verheij, M. M., Karel, P., Cools, A. R., \& Homberg, J. R. (2014). Reduced cocaine-induced serotonin, but not dopamine and noradrenaline, release in rats with a genetic deletion of serotonin transporters. Eur Neuropsychopharmacol, 24 (11), 1850-1854. doi:10.1016/j.euroneuro.2014.09.004

Verheij, M. M. M., Contet, C., Karel, P., Latour, J., van der Doelen, R. H. A., Geenen, B., . . Homberg, J. R. (2018). Median and Dorsal Raphe Serotonergic Neurons Control Moderate Versus Compulsive Cocaine Intake.Biol Psychiatry, 83 (12), 1024-1035. doi:10.1016/j.biopsych.2017.10.031

Wagner, F. A., \& Anthony, J. C. (2002). From first drug use to drug dependence; developmental periods of risk for dependence upon marijuana, cocaine, and alcohol. Neuropsychopharmacology, 26 (4), 479-488. doi:10.1016/S0893-133X(01)00367-0

Wee, S., Specio, S. E., \& Koob, G. F. (2007). Effects of dose and session duration on cocaine selfadministration in rats. J Pharmacol Exp Ther, 320 (3), 1134-1143. doi:10.1124/jpet.106.113340

Wolf, M. E. (2016). Synaptic mechanisms underlying persistent cocaine craving. Nat Rev Neurosci, 17 (6), 351-365. doi:10.1038/nrn.2016.39

Won, S., Levy, J. M., Nicoll, R. A., \& Roche, K. W. (2017). MAGUKs: multifaceted synaptic organizers. Curr Opin Neurobiol, 43 , 94-101. doi:10.1016/j.conb.2017.01.006

Woolverton, W. L., \& Johnson, K. M. (1992). Neurobiology of cocaine abuse. Trends Pharmacol Sci, 13 (5), 193-200. doi:10.1016/0165-6147(92)90063-c

Figure 1
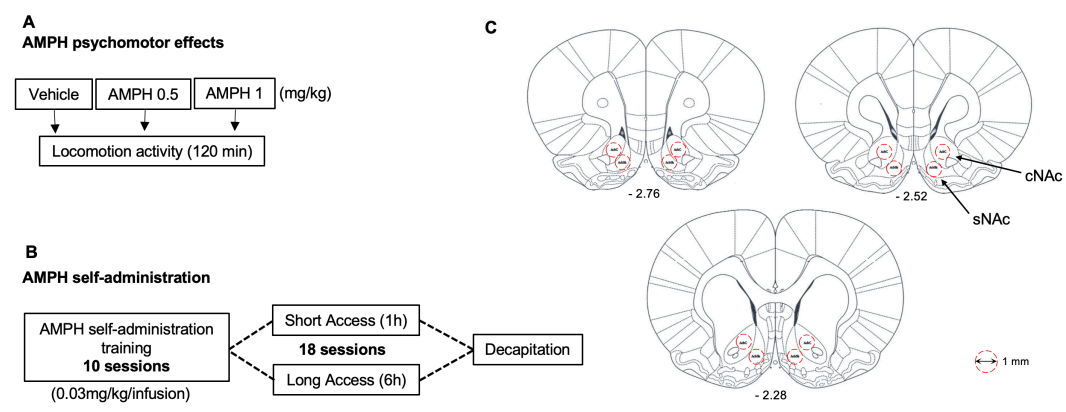


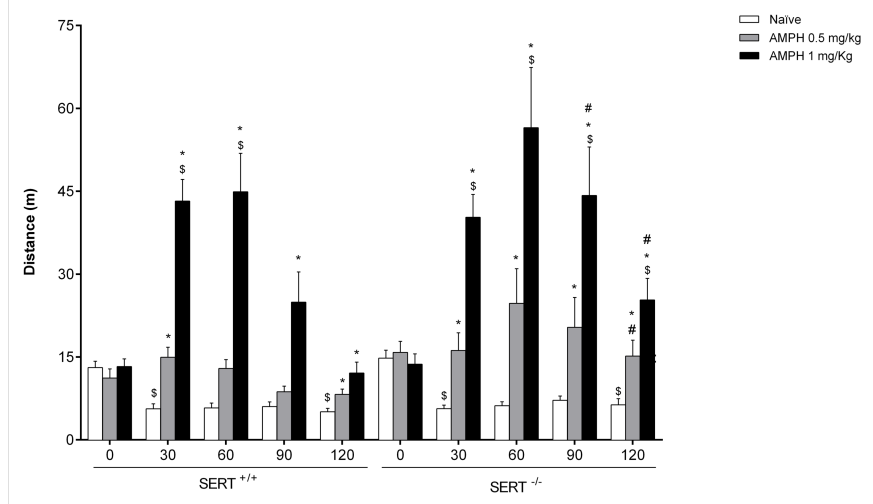

Figure 2
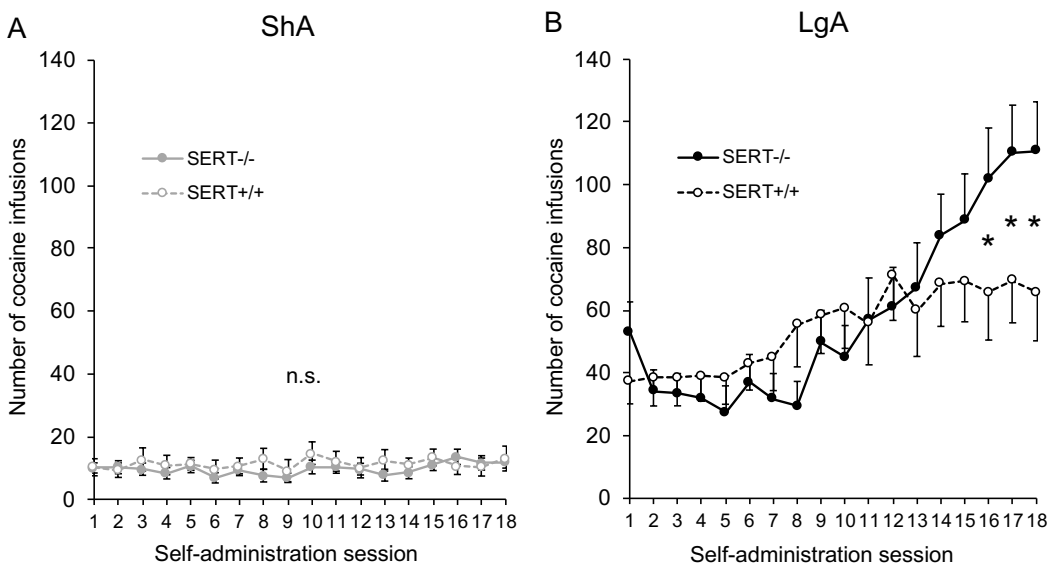

Figure 3 
Figure 4
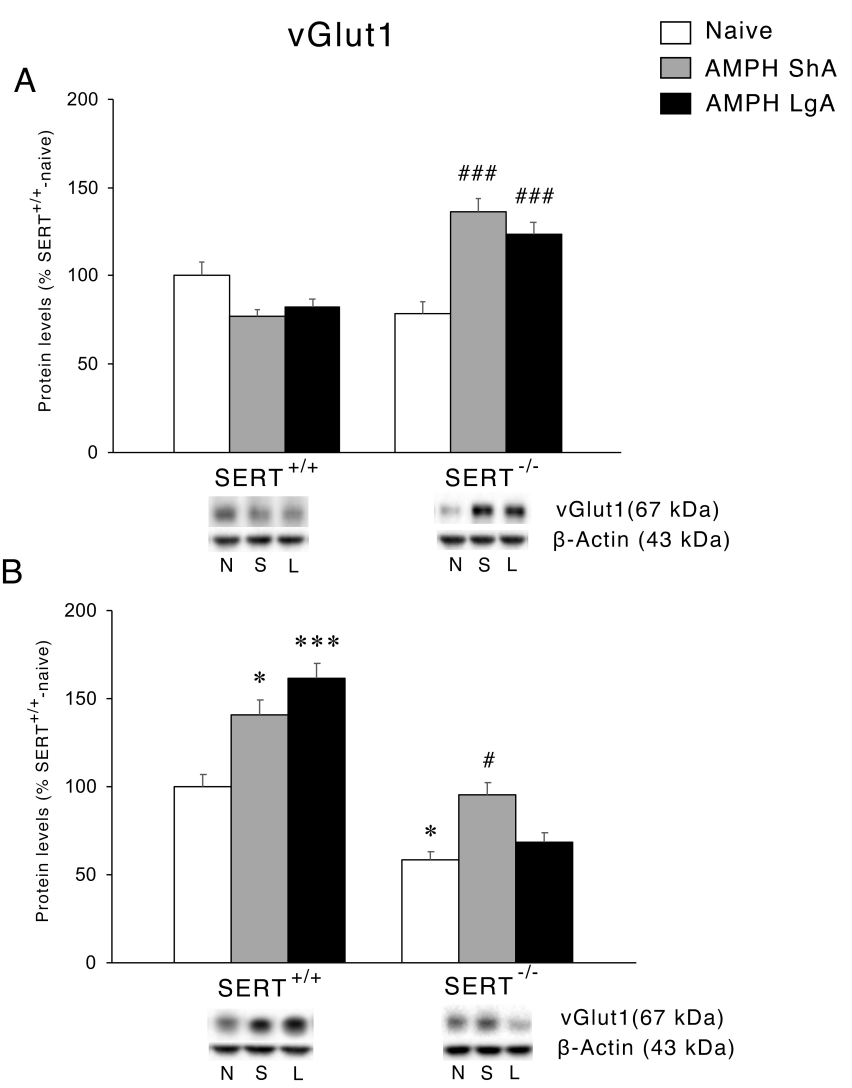
Figure 5
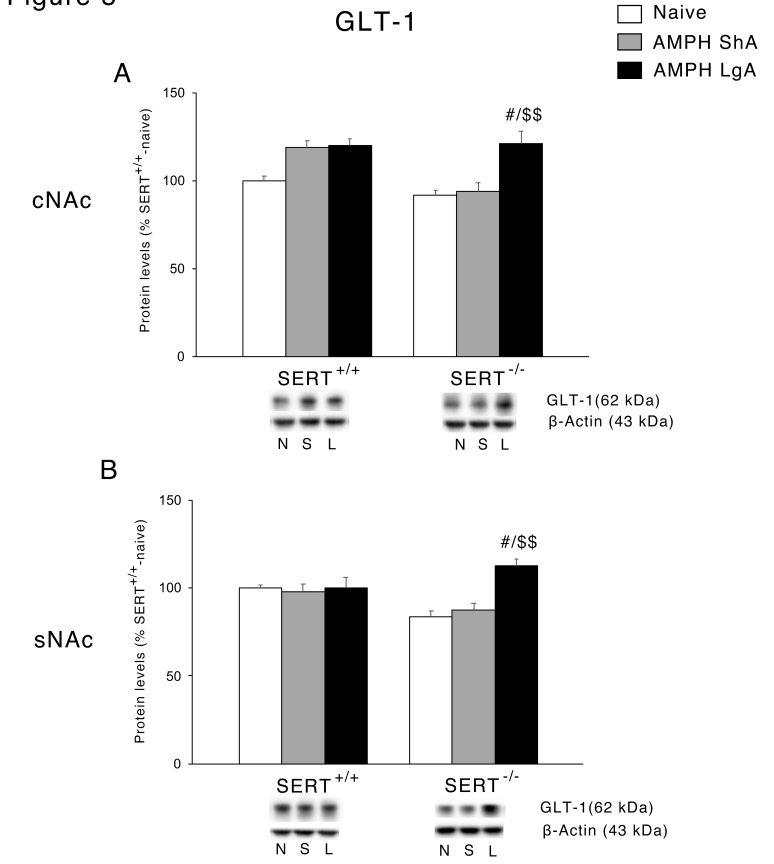

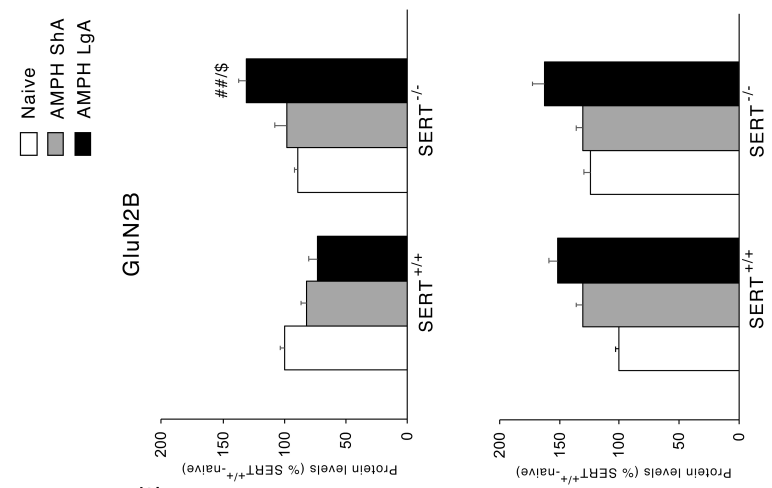

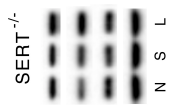

ш

ᄂ

政

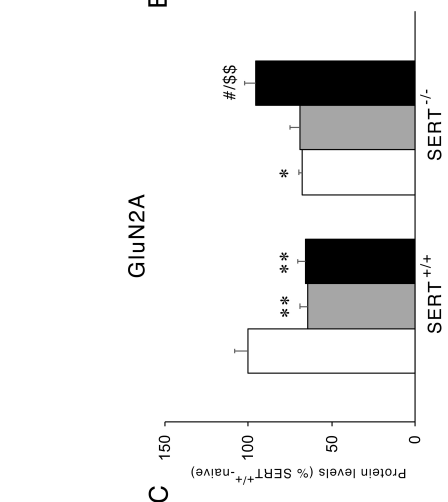

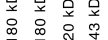

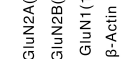

$\stackrel{+}{+}|1| 1 \mid$

点 $1111: 0$

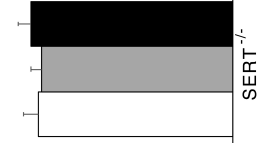

$\sum_{\infty}^{0}$

1113

䍃 $111 ; \infty$

क $1111=$

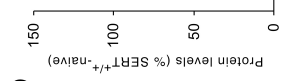

즐

口
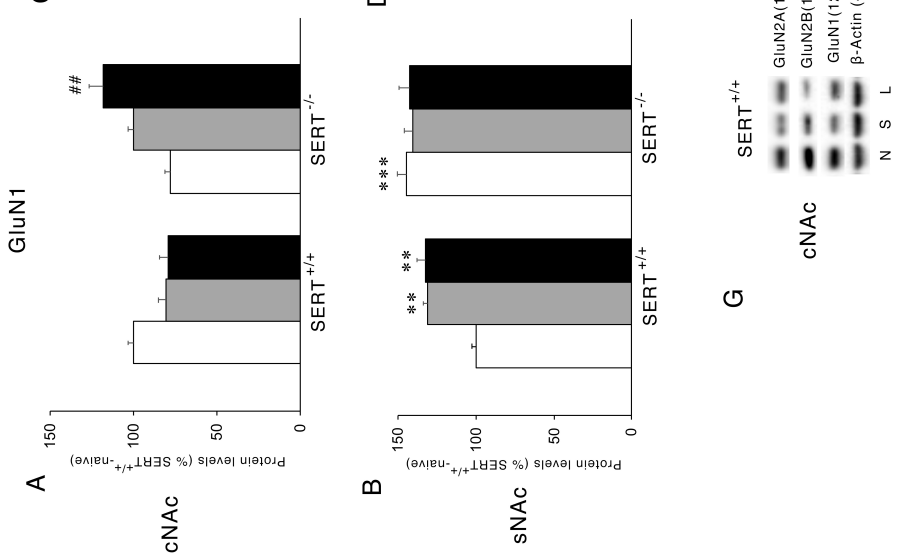
Figure 7

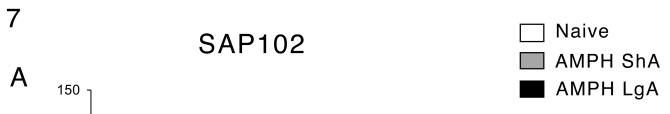

CNAC
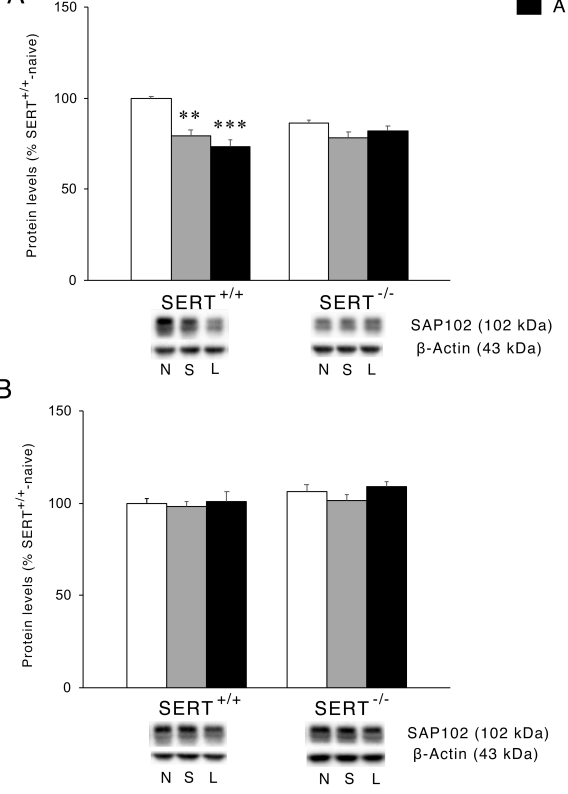

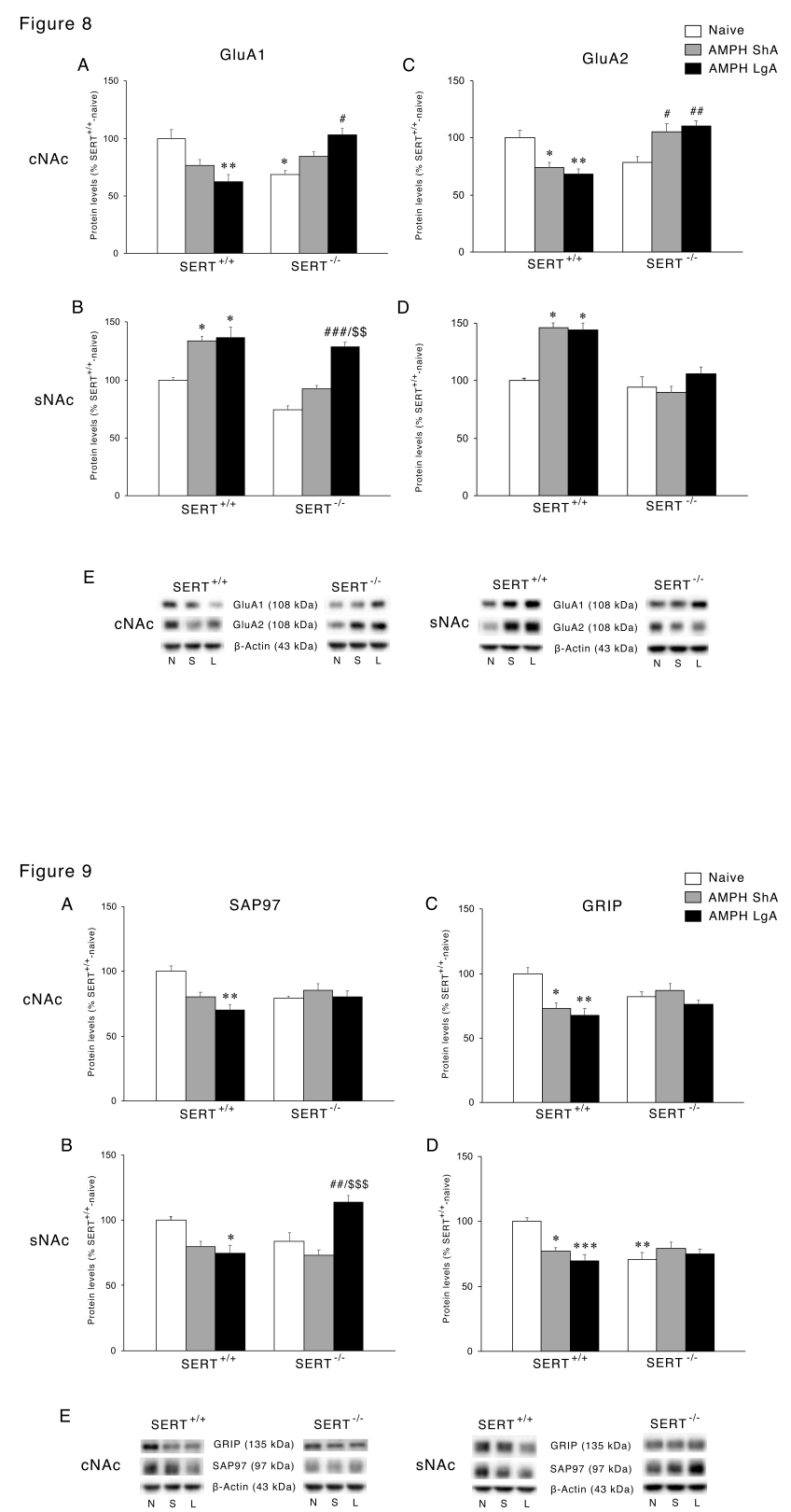
Figure 10

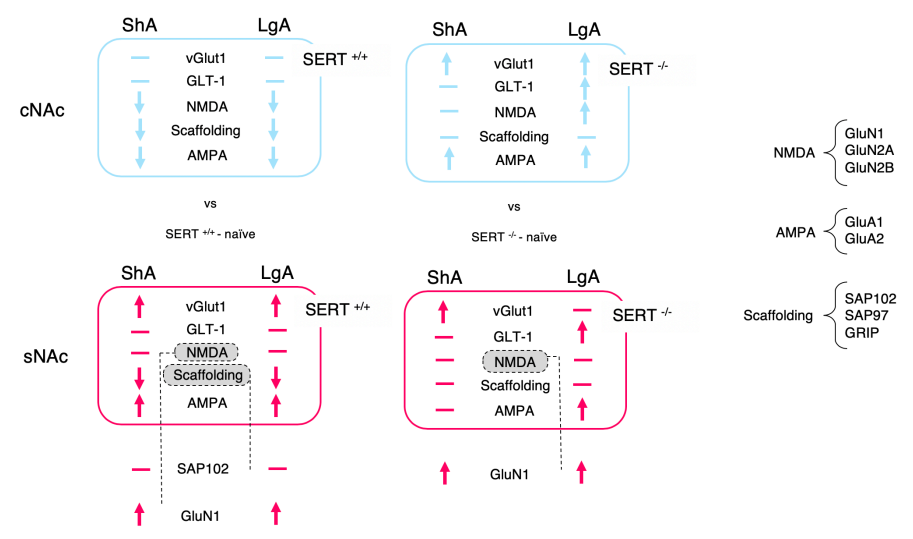

